Notre Dame Journal of Formal Logic

Volume XIV, Number 1, January 1973

NDJFAM

\title{
ON A PROPERTY OF CERTAIN PROPOSITIONAL FORMULAE
}

\author{
DAVID MEREDITH
}

In [1] section 4 Lukasiewicz gives a theorem concerning the law of syllogism. The present paper presents a much more general theorem from which the Eukasiewicz theorem can be derived. Sections 1 and 2 present our theorem; a brief discussion of its application and its relationship to the Eukasiewicz theorem is given in section 3.

1. Preliminaries and Statement of Theorem. We use ' $P$ ', ' $Q$ ', ' $R$ ', with and without subscripts to denote well-formed propositional formulae. ' $\left\{P_{1}, \ldots\right.$ $\left.P_{n}\right\}$ ', ' $\left\{Q_{1}, \ldots Q_{m}\right\}$ ' and so on denote ordered sets of such formulae. ' $\Phi$ ' is used for a constant operation under the substitution rule; ' $\Phi^{n}$ ' denotes $n$ repetitions of the operation; ' $\Phi\left\{P_{1}, \ldots P_{n}\right\}$ ' is an abbreviation for ' $\left\{\Phi P_{1}, \ldots\right.$. $\left.\Phi P_{n}\right\}$ '. ' $\cup$ ' and ' $\subset$ ' have their usual meanings. We use ' $\sim$ ' to denote a relationship between an ordered set of propositional formulae and a single formula which is defined as follows.

Definition $\left\{P_{1}, \ldots P_{n}\right\} \sim Q$ is defined inductively in two steps:

a. Let $Q$ be a member of $\left\{P_{1}, \ldots P_{n}\right\}$ : then $\left\{P_{1}, \ldots P_{n}\right\} \sim Q$.

b. For some $R$, let $\left\{P_{1}, \ldots P_{n}\right\} \sim R$ and let $\left\{P_{1}, \ldots P_{n}\right\} \sim C R Q$ : then $\left\{P_{1}, \ldots P_{n}\right\} \sim Q$.

Less formally, our relationship holds between a formula and any ordered set of formulae of which it is a member, or from which it can be obtained by one or more applications of Modus Ponens. Our theorem can now be stated.

Theorem For any well-formed formula of the form $C P_{1} \ldots C P_{n} C Q_{1} \ldots$ $C Q_{m-1} Q_{m}(m, n \geq 1)$ if the following three conditions are satisfied:

a. $\Phi C Q_{1} \ldots C Q_{m-1} Q_{m}=C P_{1} \ldots C P_{n} C Q_{1} \ldots C Q_{m-1} Q_{m}$

b. $Q_{m}$ is elementary

c. $\left\{P_{1}, \ldots P_{n}, Q_{1}, \ldots Q_{m-1}\right\} \sim Q_{m}$

then

$$
\Phi\left\{P_{1}, \ldots P_{n}\right\} \cup \Phi^{2}\left\{P_{1}, \ldots P_{n}\right\} \cup \ldots \Phi^{m}\left\{P_{1}, \ldots P_{n}\right\} \sim C P_{1} \ldots
$$


To illustrate the theorem we may take $C P_{1} \ldots C P_{n} C Q_{1} \ldots C Q_{m-1} Q_{m}=$ $C C p C q r C q C p r$, and $\Phi=p|q, q| C p C q r, r \mid C p r$. The three conditions of the hypothesis are satisfied:
a. $\Phi C q C p r=C C p C q r C q C p r$
b. $Q_{3}=r$ is elementary
c. $\{C p C q r, q, p\} \sim r$

Hence by our theorem $C C p C q r C q C p r$ is closed by the union of the following sets:

$$
\begin{aligned}
\Phi\{C p C q r\} & =C q C C p C q r C p r \\
\Phi^{2}\{C p C q r\} & =C C p C q r C C q C C p C q r C p r C q C p r \\
\Phi^{3}\{C p C q r\} & =C C q C C p C q r C p r C C C p C q r C C q C C p C q r C p r C q C p r C C p C q r C q C p r
\end{aligned}
$$

The reader can easily verify that this is so by noting that:

$$
\Phi^{3} C p C q r=C \Phi C p C q r C \Phi^{2} C p C q r C C p C q r C q C p r
$$

2. Proof of Theorem.* Before proceeding to the proof of our theorem we give five lemmas.

Lemma 1 For $m<n, \Phi C P_{1} \ldots C P_{n-1} P_{n}=C \Phi P_{1} \ldots C \Phi P_{m} \Phi C P_{m+1} \ldots$ $C P_{n-1} P_{n}$.

Lemma 2 If $\left\{P_{1}, \ldots P_{n}\right\} \sim Q$, then $\Phi^{m}\left\{P_{1}, \ldots P_{n}\right\} \sim \Phi^{m} Q$.

Lemma 3 If $\Phi\left\{P_{1}, \ldots P_{n}\right\}=\left\{Q_{1}, \ldots Q_{m}\right\}$, then $\Phi^{l}\left\{P_{1}, \ldots P_{n}\right\}=\Phi^{l-1}\left\{Q_{1}\right.$, ... $\left.Q_{m}\right\}$.

Proof. This follows from the lemma's hypothesis in virtue of the fact that $\Phi^{l}\left\{P_{1}, \ldots P_{n}\right\}=\Phi^{l-1} \Phi\left\{P_{1}, \ldots P_{n}\right\}$.

Lemma 4 If $\Phi C Q_{1} \ldots C Q_{m-1} Q_{m}=C P_{1} \ldots C P_{n} C Q_{1} \ldots C Q_{m-1} Q_{m}(m, n \geq 1)$, then for $k n-n+l \leq m-1$ where $k \geq 1$ and $l \leq n$

$$
\Phi^{k}\left\{Q_{k n-n+1}, \ldots Q_{k n-n+l}\right\}=\left\{P_{1}, \ldots P_{l}\right\} \text {. }
$$

Proof. We assume that the lemma's hypothesis is satisfied. Then by Lemma 1 we have

(1) $\Phi\left\{Q_{1}, \ldots Q_{l}\right\}=\left\{P_{1}, \ldots P_{l}\right\}$

(2) $\Phi\left\{Q_{n+1}, \ldots Q_{n+l}\right\}=\left\{Q_{1}, \ldots Q_{l}\right\}$.

From (2) by Lemma 3 and the hypothesis, we have

(3) $\Phi^{k}\left\{Q_{k n-n+1}, \ldots Q_{k n-n+l}\right\}=\Phi^{k-1}\left\{Q_{(k-1) n-n+1}, \ldots Q_{(k-1) n-n+l}\right\}$

Our lemma follows from (3) and (1).

Lemma 5 If $\Phi C Q_{1} \ldots C Q_{m-1} Q_{m}=C P_{1} \ldots C P_{n} C Q_{1} \ldots C Q_{m-1} Q_{m}(m, n \geq 1)$ then proof.

*The author is indebted to Lars Svenonius for help given him in 1957 with this 


$$
\Phi^{l} C Q_{1} \ldots C Q_{m-1} Q_{m}=C R_{1} \ldots C R_{k} C P_{1} \ldots C P_{n} C Q_{1} \ldots C Q_{m-1} Q_{m}
$$

$(l>1, k \geq 1)$ where $\left\{R_{1}, \ldots R_{k}\right\} \subset \Phi\left\{P_{1}, \ldots P_{n}\right\} \cup \ldots \Phi^{l-1}\left\{P_{1}, \ldots P_{n}\right\}$.

Proof. We assume the lemma's hypothesis. Then by Lemma 1 we have

(1) $\Phi^{l} C Q_{1} \ldots C Q_{m-1} Q_{m}=C \Phi^{l-1} P_{1} \ldots C \Phi^{l-1} P_{n} \Phi^{l-1} C Q_{1} \ldots C Q_{m-1} Q_{m}$.

Our lemma follows from (1) and the hypothesis.

To prove our theorem we assume that conditions (a) through (c) of the hypothesis are satisfied. From Lemma 4 we have:

(1) $\Phi^{k+1}\left\{Q_{k n-n+1}, \ldots Q_{k n-n+l}\right\}=\Phi\left\{P_{1}, \ldots P_{l}\right\}$

and hence by the meaning of $\Phi\left\{P_{1}, \ldots P_{n}\right\}$,

(2) $\Phi^{k+1} Q_{k n-n+l}=\Phi P_{l}$.

By the hypothesis of Lemma $4, k n-n+l \leq m-1$. Hence for $n=1, k \leq$ $m-1$ and $k+1 \leq m$. Therefore from (2) we have

(3) $\Phi^{m} Q_{m-1}=\Phi^{m-k} P_{l}$.

Purely from the meaning of the symbols involved, we can assert

(4) $\begin{aligned} & \Phi^{m}\left\{P_{1}, \ldots P_{n}, Q_{1}, \ldots Q_{m-1}\right\}=\Phi^{m}\left\{P_{1}, \ldots P_{n}\right\} \cup \Phi^{m}\left\{Q_{1}, \ldots Q_{n}\right\} \cup \ldots \\ & \Phi^{m}\left\{Q_{k n-n+1}, \ldots Q_{k n-n+l}\right\}\end{aligned}$

for $k n-n+l \leq m-1$ where $k \geq 1$ and $l \leq n$. From (4) by Lemma 4 and (3) it follows that

(5) $\Phi^{m}\left\{P_{1}, \ldots P_{n}, Q_{1}, \ldots Q_{m-1}\right\}=\Phi^{m}\left\{P_{1}, \ldots P_{n}\right\} \cup \Phi^{m-1}\left\{P_{1}, \ldots P_{n}\right\} \cup \ldots$ $\Phi^{m-k}\left\{P_{1}, \ldots P_{l}\right\}$

and hence we derive

(6) $\Phi^{m}\left\{P_{1}, \ldots P_{n}, Q_{1}, \ldots Q_{m-1}\right\} \subset \Phi\left\{P_{1}, \ldots P_{n}\right\} \cup \ldots \Phi^{m}\left\{P_{1}, \ldots P_{n}\right\}$.

From condition (c) of the hypothesis, we have, by Lemma 2

(7) $\Phi^{m}\left\{P_{1}, \ldots P_{n}, Q_{1}, \ldots Q_{m-1}\right\} \sim \Phi^{m} Q_{m}$.

From (6) and (7) it follows that

(8) $\Phi\left\{P_{1}, \ldots P_{n}\right\} \cup \ldots \Phi^{m}\left\{P_{1}, \ldots P_{n}\right\} \sim \Phi^{m} Q_{m}$.

We now turn attention to $\Phi^{m} Q_{m}$. By condition (a) of the hypothesis we have

(9) a. $\Phi Q_{m}=C Q_{m-n} \ldots C Q_{m-1} Q_{m}$ when $n<m-1$

b. $\Phi Q_{m}=C Q_{1} \ldots C Q_{m-1} Q_{m}$ when $n=m-1$

c. $\Phi Q_{m}=C P_{m} \ldots C P_{n} C Q_{1} \ldots C Q_{m-1} Q_{m}$ when $n>m-1$.

From (9) by condition (a) again and Lemma 1, we derive

(10) where $l$ is the least integer such that $l n \geq m-1$
a. $\Phi^{l+1} Q_{m}=C P_{1} \ldots C P_{n} C Q_{1} \ldots C Q_{m-1} Q_{m}$ when $l n=m-1$
b. $\Phi^{l+1} Q_{m}=C \Phi P_{n-l n+m} \ldots C \Phi P_{n} C P_{1} \ldots C P_{n} C Q_{1} \ldots C Q_{m-1} Q_{m}$ when $\ln >$ $m-1$. 
By the hypothesis in (10) $m \geq l+1$. Further $\Phi\left\{P_{n-l n+m} \ldots P_{n}\right\} \subset \Phi\left\{P_{1}\right.$, $\left.\ldots P_{n}\right\}$. Therefore from (10) by Lemma 5 it follows that

(11) a. $\Phi^{m} Q_{m}=C P_{1} \ldots C P_{n} C Q_{1} \ldots C Q_{m-1} Q_{m}$ or

b. $\Phi^{m} Q_{m}=C R_{1} \ldots C R_{k} C P_{1} \ldots C P_{n} C Q_{1} \ldots C Q_{m-1} Q_{m}$

where $\left\{R_{1}, \ldots R_{k}\right\} \subset \Phi\left\{P_{1}, \ldots P_{n}\right\} \cup \ldots \Phi^{m-l-1}\left\{P_{1}, \ldots P_{n}\right\}(k \geq 1)$. From (11) by the definition of $\sim$, it follows that

(12) $\Phi\left\{P_{1}, \ldots P_{n}\right\} \cup \ldots \Phi^{m-l-1}\left\{P_{1}, \ldots P_{n}\right\} \cup\left\{\Phi^{m} Q_{m}\right\} \sim C P_{1} \ldots C P_{n} C Q_{1} \ldots$ $C Q_{m-1} Q_{m}$.

In virtue of the fact that $\Phi\left\{P_{1}, \ldots P_{n}\right\} \cup \ldots \Phi^{m-l-1}\left\{P_{1}, \ldots P_{n}\right\} \subset \Phi\left\{P_{1}, \ldots\right.$ $\left.P_{n}\right\} \cup \ldots \Phi^{m}\left\{P_{1}, \ldots P_{n}\right\}$ our theorem follows from (8) and (12).

3. Application of Theorem. Our theorem is useful for discovering derivations for formulae within Propositional Calculus. One example is the by no means obvious derivation of $C C p C q r C q C p r$ from $C q C C p C q r C p r$ given in section 1. Another is given by taking $C P_{1} \ldots C P_{n} C Q_{1} \ldots C Q_{m-1} Q_{m}=$ $C C p q C C q r C p r$, and $\Phi=p|C p q, q| \alpha, r \mid C C q r C p r$. By our theorem $\Phi\{C p q$, $C q r\} \cup \Phi^{2}\{C p q, C q r\} \sim C C p q C C q r C p r$. The union yields the four formulae
(1) $c C p q \alpha$
(2) $C \alpha C C q r C p r$
(3) $C \subset p q \alpha \Phi \alpha$
(4) $C \Phi \alpha C C \alpha C C q r C p r C C p q C C q r C p r$

From (1) and (3) we get $\Phi \alpha$ by Modus Ponens. With $\Phi \alpha$ and (2), two applications of Modus Ponens to (4) yield the original formula. This instance of our theorem is a proof of the Eukasiewicz theorem referred to above, which states that from any two formulae of the form (1) and (2) the law of syllogism can be derived using only Substitution and Modus Ponens. Useful derivations result when $\alpha$ is so chosen that CCpq $\alpha$ and $C \alpha C C q r C p r$ are theses.

\section{REFERENCES}

[1] Eukasiewicz, Jan, "The Shortest Axiom of the Implicational Calculus of Propositions," Proceedings of the Royal Irish Academy, vol. 52, Sec. A, No. 3 (1948). 